\title{
Aerial Data Aggregation in IoT Networks: Hovering \& Traveling Time Dilemma
}

\author{
Osama M. Bushnaq ${ }^{\dagger}$, Abdulkadir Celik ${ }^{\dagger}$, Hesham ElSawy ${ }^{\ddagger}$, Mohamed-Slim Alouini $^{\dagger}$, Tareq Y. Al-Naffouri ${ }^{\dagger}$ \\ ${ }^{\dagger}$ Computer, Electrical and Mathematical Siences and Engineering (CEMSE) Division \\ King Abdullah University of Science and Technology, Thuwal, Saudi Arabia \\ ${ }^{\ddagger}$ Department of Electrical Engineering, King Fahd University of Petroleum \& Minerals, Dhahran, Saudi Arabia
}

\begin{abstract}
The next era of information revolution will rely on aggregating big data from massive numbers of devices that are widely scattered in our environment. The majority of these devices are expected to be of low-complexity, low-cost, and limited power supply, which impose stringent constraints on the network operation. In this regards, this paper proposes aerial data aggregation from a finite spatial field via an unmanned aerial vehicle (UAV). Instead of fusing, relaying, and routing the data across the wireless nodes to fixed locations access points, an UAV flies over the field and collects the required data. Particularly, the field is divided into several subregions over which the UAV hovers to collect samples from the underlying nodes. To this end, an optimization problem is formulated and solved to find the optimal number of subregions, the area of each subregion, the hovering locations, the hovering time at each location, and the trajectory traversed between hovering locations such that an average number of samples are collected from the field in minimal time. The proposed formulation is shown to be np-hard mixed integer problem, and hence, a decoupled heuristic solution is proposed. The results show that there exists an optimal number of subregions that balance the tradeoff between hovering and traveling times such that the total time for collecting the required samples is minimized.
\end{abstract}

Keywords-Unmanned aerial vehicle (UAV); Internet of things (IoT); stochastic geometry; coverage problem, travel salesman.

\section{INTRODUCTION}

The Internet of Things (IoT) is a foundational building block for the upcoming information revolution and imminent smart-world era. Particularly, the IoT bridges the cyber domain to everything and anything within our physical world (e.g., goods, appliances, vehicles, light poles, parking meters, plants, etc.), which enables unprecedented ubiquitous monitoring and smart control. For a cost effective materialization of such vision, the IoT relies on low-cost wireless sensor nodes with short transmission ranges, limited energy supply, and constrained computational capabilities [1]. The high density and wide-spatial distribution of the IoT sensors along with the stringent operational constraints for each sensor render the conventional data aggregation schemes (i.e., clustering and multi-hopping) obsolete [2].

Exploiting the significant advances in global unmanned aerial vehicle (UAV) market, flying data aggregators can be used to collect the IoT big data [3]. Instead of disseminating

This work is supported by the KAUST-MIT-TUD consortium under grant OSR-2015-Sensors-2700. and routing the data across the network to fixed location access points, UAVs with wireless communication capabilities fly across the network to collect the data. This offloads the data aggregation burden from the IoT sensors to the UAVs, which offers multifold gains for the IoT implementation and operation. For instance, recharging and maintaining the UAVs that eventually fly back to central headquarters impose much less overhead than recharging and maintaining, respectively, the sensors and access points in the field. The sensors are not responsible of data relaying and routing, and hence, simpler and lower-cost sensors can be utilized. On demand wake-up schemes upon data collection can be utilized, which reduces energy consumption and prolongs the network life time.

The recent advancement, along with the reduced cost, of UAVs have attracted much interest from the information and communication technology (ICT) industry. For instance, flying base stations can be used to improve cellular coverage, provide wireless communications during natural disasters and public safety operations, and extend wireless services for rural areas [4], [5]. The surging UAV use cases within the ICT motivated the research community to model, assess, and develop design paradigms for hybrid terrestrial/airborne communication systems. For instance, the studies in [6][10] characterize signal propagation and fading over airto-ground (A2G) and ground-to-air (G2A) communications links. Coverage probability and communication rates for UAV downlink networks are derived in [11]. An optimization framework for the positions and hover times for multiple UAVs serving downlink and uplink users are proposed in [12] and [13], respectively. However,the focus in [11]-[13] is on the airborne to terrestrial coverage problems. In the context of uplink data aggregation, the throughput per pass for a UAV over a sensor network is characterized in [14]. Trajectory and speed optimization framework for data aggregation from sensor networks are provided in [15]. However, the studies in [14], [15] are for fixed-wing UAVs (i.e., no hovering capability). To the best of the authors knowledge, the problem of data aggregation with rotary-wings (i.e., hovering capable) UAV from a large-scale IoT network has not been considered in the literature.

This paper proposes aerial data aggregation from an IoT network that is scattered according to a Poisson point process (PPP) over a finite field. Exploiting the hovering capability of the UAV, reliable transmissions of data can be attained by 


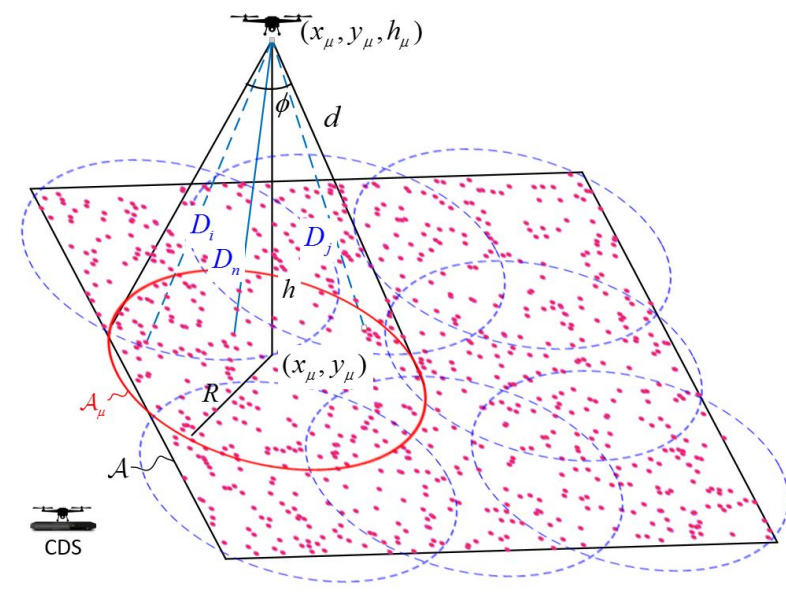

Fig. 1: System model

activating the nodes when the UAV is stationary. Particularly, the field is divided into several subregions where the UAV sequentially hovers over each subregion to collect data from the underlying nodes. At each hovering location, the UAV sends a universal activation/synchronization signal to all nodes covered from that hovering location. The activated nodes then sends back their data via slotted ALOHA protocol. To this end, an optimization problem is formulated and solved to find the optimal number of subregions, the area of each subregion, the hovering locations, the hovering time at each location, and the trajectory between hovering locations such that an average number of samples are collected from the field in minimal time. The main contribution of the paper are as follows

- An optimization problem is formulated and solved to minimize the time for aggregating an average number of samples from a finite field using a rotary-wing UAV.

- Stochastic geometry is utilized to characterize the transmission success probability of data while accounting for the spatial randomness and Nakagami-m channel fading.

- A simple feasible solution for minimizing the total data aggregation is presented. The developed solution takes into consideration the physical and mechanical constraints related to the UAV acceleration, deceleration, and top speed.

The paper is organized as follows. In Section II, the system model is described. In Section III, the optimization problem is stated, formulated and discussed. In Sections IV and V, the hovering and traveling times are derived which then leads to a suboptimal solution. In Section VI, analytical and simulation results are provided. Finally, we provide our conclusions in Section VII.

\section{SYSTEM MODEL}

We consider a dense IoT network that is confined within a finite region $\mathcal{A}$. The IoT devices are distributed in $\mathcal{A}$ according to a homogeneous PPP $\Psi$ with density $\lambda$. An $\mathrm{UAV}$, popularly known as a drone, is equipped as an airborne base station to collect data samples (e.g., field monitoring) from the IoT nodes every time it flies over $\mathcal{A}$. The employed drone knows the boundaries of the field $\mathcal{A}$ but is oblivious to the exact locations and number of the IoT nodes within $\mathcal{A}$. Hence, the objective is to fly over $\mathcal{A}$ such that an average of $\zeta$ samples, diversified as much as possible, are collected in minimal time.

For each samples collection trip, the drone leaves its charging-docking station (CDS), goes to $M$ different hovering-locations (HLs) within $\mathcal{A}$, and finally returns back to the CDS. The drone stays at each HL for a deterministic time duration, denoted as the hovering time, to collect samples from the covered nodes. Fig. 1 shows the network model, the HLs for $M=8$, and the circular sub-regions covered from each HL. ${ }^{1}$ Note that the drone's altitude $h$ for a given $M$ is determined by the radius of the circles and the antenna beam-width $\phi$ as $R=h \tan (\phi / 2)$.

Given the spatial homogeneity of the IoT network, the drone stays for $T_{\text {hover }}$ at each HL such that an average of $\frac{\zeta}{M}$ samples is collected, which diversifies the acquired samples from $\mathcal{A}$. Let $\boldsymbol{L}=\left[\boldsymbol{\ell}_{\boldsymbol{1}}, \ldots, \boldsymbol{\ell}_{\boldsymbol{\mu}}, \ldots, \boldsymbol{\ell}_{\boldsymbol{M}}\right]^{T} \in \mathbb{R}^{M \times 3}$ denote all the HLs where $\ell_{\mu} \in \mathbb{R}^{3}$ is the $3 \mathrm{D}$ coordinates of the $\mathrm{HL}_{\mu}$, $\mu \in\{1,2, \ldots, M\}$. At each HL, the covered circular region is denoted as $\mathcal{A}_{\mu}\left(x_{\mu}, y_{\mu}, R\right)$ with an area of $\left|\mathcal{A}_{\mu}\right|=\pi R^{2}$, where $|\cdot|$ denotes the Lebesgue measure. For a given $M$, the radius $R$ and the hovering locations $\boldsymbol{L}$ are selected such that the union of all sub-regions encompasses the entire network, i.e., $\mathcal{A} \subseteq \bigcup_{\mu} \mathcal{A}_{\mu}$ (cf. Fig. 2). Furthermore, the trajectory between the HLs is selected such that the total distance traveled by the drone is minimized. It is worth noting that the drone does not move with a uniform velocity between the HLs. Instead, due to mechanical and physical constrains, the drone accelerates with rate $\hat{q}\left[\mathrm{~m} / \mathrm{s}^{2}\right]$ and then decelerates with rate $\breve{q}\left[\mathrm{~m} / \mathrm{s}^{2}\right]$ between any two stationary HLs, where the acceleration saturates at the drone maximum speed of $\nu[\mathrm{m} / \mathrm{s}]$. The transition from acceleration to deceleration is initiated such that the drone becomes stationary (i.e., reaches zero velocity) at the target HL.

\section{A. Data Aggregation Model}

During each hovering epoch, the drone first broadcasts a signal to activate and synchronize nodes within $\mathcal{A}_{\mu}$. The activated nodes transmit over a common radio spectrum using a slotted ALOHA medium access scheme with transmission probability $a$. The nodes operate at a fixed rate of $\log _{2}(1+$ $\beta$ ) $[$ bits $/ \mathrm{Hz} / \mathrm{s}]$, and hence, a transmission is successful if the signal-to-interference-plus-noise-ratio (SINR) at the drone is greater than $\beta$. At a given transmission instant, all activated nodes that are elected by the ALOHA protocol mutually interfere with each other. As mentioned before, the objective of the drone is to collect an average of $\zeta$ samples from $\mathcal{A}$ irrespective of the transmitting nodes identities. Hence, the data sent by the node with the maximum SINR is treated as the intended transmission and all other transmissions are considered interference.

\footnotetext{
${ }^{1}$ The areas of all sub-regions, as well as the hovering time at each HL, are designed to be equal due to the spatial homogeneity of the IoT network. Otherwise, the area of each subregion (or the hovering time at each HL) should be proportional to the spatial density of the covered IoT devices.
} 
Transmissions over the ground-to-Air (G2A) links experience power-law path-loss attenuation and Nakagami- $m$ multi-path fading. That is, the signal power decays at a rate of $D^{-\eta}$ with the distance $D$, where $\eta$ is the path loss exponent. Furthermore, the power of each transmitted signal experience independent and identically distributed Gamma distributed gain, denoted as $G$, with the following probability density function (PDF) [16]

$$
f_{G}(g)=\frac{m^{m} g^{m-1}}{\Gamma(m)} \exp (-m g),
$$

where $\Gamma(\cdot)$ is the gamma function. Setting $m=1$ models the well-known non-line of sight Rayleigh $(m=1)$ fading environment. Setting $m>1$ approximates the light of sight Rician fading [17]. For the sake of analytical tractability, the parameter $m$ is selected to be an integer, $m \in \mathbb{Z}^{+}$. According to the aforementioned system model, the SINR for an arbitrary selected active node is given by

$$
\operatorname{SINR}_{\mu}^{n}=\frac{P G_{n} D_{n}^{-\eta}}{\sum_{\mathbf{x} \in \tilde{\Psi}_{\mu} \backslash \mathbf{x}_{\mathbf{n}}} P G_{x} D_{x}^{-\eta}+\sigma^{2}},
$$

where $\tilde{\Psi}_{\mu}$ is the subset of nodes that are simultaneously transmitting, $P$ is the IoT nodes transmission power and $\sigma^{2}$ is the background noise variance. Note that the IoT nodes are typically manufactured with stringent power budgets, and hence, the noise variance $\sigma^{2}$ has a paramount influence on the SINR.

\section{Hovering \& Traveling Time Dilemma}

In the realm of traditional wireless networks, data aggregation is typically designed to minimize the amount and duration of data transmission in order to maximize network lifetime and utilize the available bandwidth. For a powerhungry vehicle relying upon a limited battery capacity, such a goal is particularly of a vital importance in aerial data aggregation. The hovering time is primarily determined by the number of samples to be collected along with the wireless links quality. Traveling time between the HLs adds a second dimension to this already challenging task. This is because it is desirable to fulfill the data aggregation task as soon as possible and return to the docking station for recharging.

Therefore, our main purpose in this paper is to minimize total data aggregation time, which can be decomposed to hovering and traveling times. At this very moment, we find ourselves on the horns of the following dilemma: On the one hand, a lower $M$ yields to higher latitudes and larger subregions, which degrades the SINR due to worse link quality and higher interference. At the extreme case of $M=1$, the drone has to hover at a high altitude for a very long time to handle all traffic requests most of which have a low probability of success due to the bad channel quality and high interference. On the another hand, total traveling time increases with the number of stations. For high number of HLs, the drone cannot fully exploit its agility as shorter distances between the stations enforces the drone to decelerate before reaching its peak velocity. Note also that the drone needs some time for reconfiguration after arrivals to, and before departures from, HLs. More importantly, a high number of HLs may lead to unnecessarily small coverage regions that encompass no IoT devices, which wastes the traveling, hovering, and reconfigurations times related to such empty HLs. The formal definition of the considered aerial data aggregation problem is given as follows:

$\boldsymbol{P}_{\mathbf{1}}$ : While ensuring that the average number of observations collected from the entire network is equal to $\zeta$, we minimize the total hovering and traveling times by optimizing the following variables:

1) Number of HLs $(M)$;

2) Locations of the HLs $(\mathbf{L})$ and radius of the circular sub-regions $(R)$ such that union of the sub-regions covers all the nodes; and

3) Flight path of the drone which is characterized by $\boldsymbol{Z} \in$ $\{0,1\}^{M \times M}$ where $z_{i j}=1$ if the drone departs from $H L_{i}$ to arrive $H L_{j}$ and $z_{i j}=0$ otherwise.

The mathematical formulation to minimize the data aggregation time is given by

$$
\begin{array}{ll}
\min _{M, R, \mathbf{L}, \mathbf{Z}, \boldsymbol{\varphi}} & T_{\text {total }}=\sum_{\mu=1}^{M} T_{\text {hover }}^{\mu}+T_{\text {travel }} \\
\text { s.t. } \quad & \sum_{\mu=1}^{M} \mathbb{E}\left\{\mathrm{K}_{\mu}\right\} \geq \zeta \\
& \mathcal{A} \subseteq \bigcup_{\mu=1}^{M} \mathcal{A}_{\mu}, \\
& \sum_{i=1, i \neq j}^{M} z_{i, j}=1, \quad \forall j \in[1, M] \\
& \sum_{j=1, j \neq i}^{M} z_{i, j}=1, \quad \forall i \in[1, M] \\
& \varphi_{i}-\varphi_{j}+M z_{i, j} \leq M-1,2 \leq i \neq j \leq M
\end{array}
$$

where $T_{\text {hover }}^{\mu}$ is the hovering time at $\mathrm{HL}_{\mu}, T_{\text {travel }}$ is the traveling time, $\varphi$ is an auxiliary variable to exclude subtours in the flight paths and $\mathrm{K}_{\mu}$ is the number of successfully received observations at $\mathrm{HL}_{\mu}$. The constraint in (3a) makes sure that the drone receives on average $\zeta$ successful transmission from the entire network. In (3b), we guarantee that all nodes are covered by the union of the hovering areas. The constraint in $(3 \mathrm{c})$ ensure that each HL is visited from exactly one other HL. Likewise, (3d) assures that there is exactly one departure from each HL. In other words, $\mathbf{Z}$ has exactly one entry equals one in each row and column. Finally, (3e) eliminates the possible subroutes and known as MillerTucker-Zemlin formulation.

$\boldsymbol{P}_{\mathbf{1}}$ is a mixed-integer nonlinear programming (MINLP) problem which has impractically high computational complexity even for the moderate sized networks. Therefore, we divide this problem into hovering and traveling time minimization sub-problems for a given $M$ which is the most complicating (coupling) variable of $\boldsymbol{P}_{\mathbf{1}}$. 


\section{Hovering TIME: $T_{\text {hover }}$}

Hovering time is regulated by the chain relation of $M \rightarrow$ $(\boldsymbol{L}, R) \rightarrow \mathcal{A}_{\mu}$, that is, the coverage region for each $\mathrm{HL}$ is directly determined by $M$. Let $\Psi_{\mu}=\Psi \cap \mathcal{A}_{\mu}$ be the set of nodes covered from the HL $\ell_{\mu}$. Then, \# $\left(\Psi_{\mu}\right) \sim$ Pois $\left(\lambda\left|\mathcal{A}_{\mu}\right|\right)$, where $\#(\cdot)$ indicates the number of points within a given set. Furthermore, the location of the nodes within $\mathcal{A}_{\mu}$ are independently and uniformly distributed. Hence, the distance between a randomly selected node in $\mathcal{A}_{\mu}$ and the drone is given by

$$
f_{D}(r)=\frac{2 r}{R^{2}} \quad h_{\mu} \leq r \leq d,
$$

where $h_{\mu}$ is the UAV altitude and $d=\sqrt{h_{\mu}^{2}+R^{2}}$ is the hypotenuse of the triangle formed by $\ell_{\mu},\left(x_{\mu}, y_{\mu}\right)$, and the edge of $\mathcal{A}_{\mu}$. The angle between the hypotenuse and height cathetus is denoted by $\phi / 2$ (Fig. 1).

The pair $(\boldsymbol{L}, R)$ has a significant impact on SINR levels and probability of successful transmissions, which governs the hovering time to receive a target average number of successful transmissions. Assuming the ALOHA protocol for the medium access, each node independently accesses the channel and transmits to the drone with probability $a$. Let $\tilde{\Psi}_{\mu} \subseteq \Psi_{\mu}$ be the subset of nodes that are simultaneously transmitting to the drone at a given time instant. Then, $\tilde{\Psi}_{\mu}$ is a PPP with intensity function $\lambda(\mathbf{x})=a \lambda \mathbb{1}_{\left\{\mathbf{x} \in \mathcal{A}_{\mu}\right\}}$, where $\mathbb{1}_{\{\cdot\}}$ is the indicator function that takes the value 1 if the statement $\{\cdot\}$ is true and zero otherwise. A successful sample is collected by the drone from the subset $\tilde{\Psi}_{\mu}$ if $\#\left(\tilde{\Psi}_{\mu}\right)>0$ and the received SINR at the drone is greater than $\beta$. Note that $\beta \geq 1$ implies that at most one node from $\tilde{\Psi}_{\mu}$ can satisfy the SINR threshold [18]. Accordingly, we derive the probability of successful sample collection, denoted hereafter as the success probability, in the following theorem

Theorem 1: For a given SINR threshold $\beta \geq 1$ and ALOHA transmission probability $a$, the success probability for a transmission from $\mathcal{A}_{\mu}$ over the G2A Nakagami-m fading channel is given by

$$
\begin{aligned}
& P_{\mu}^{s}=\mathbb{P}\left(\bigcup_{\mathbf{x}_{\mathbf{n}} \in \tilde{\mathbf{\Psi}}_{\mu}} \operatorname{SINR}_{\mu}^{\mathrm{n}} \geq \beta\right)=\mathbb{E}\left\{\sum_{\mathbf{x}_{\mathbf{n}} \in \tilde{\mathbf{\Psi}}_{\mu}} \mathbb{1}_{\left\{\mathrm{SINR}_{\mu}^{\mathrm{n}} \geq \beta\right\}}\right\} \\
& =2 a \pi \lambda \int_{h}^{d} \sum_{k=0}^{m-1} \frac{\left(-m \beta r^{\eta}\right)^{k}}{k !}\left[\frac{\partial^{k}}{\partial s^{k}} \mathcal{L}_{I}(s)\right]_{s=m \beta r \eta} r d r,
\end{aligned}
$$

where $\mathrm{SINR}_{\mu}^{\mathrm{n}}$ is the SINR when $\mathbf{x}_{\mathbf{n}}$ is the intended transmitter and all other nodes in $\tilde{\Psi}_{\mu} \backslash \mathbf{x}_{\mathbf{n}}$ are interferers, $\frac{\partial^{k}}{\partial s^{k}}$ is the $k$-th partial derivative with respect to $s$, and $\mathcal{L}_{I}(s)$ is the Laplace transform of the normalized interference-plus-noise power distribution, $I=\sum_{\mathbf{x} \in \tilde{\mathbf{\Psi}}_{\mu} \backslash \mathbf{x}_{\mathbf{n}}} G_{x} R_{x}^{-\eta}+\sigma^{2} / P$, which is expressed as

$$
\begin{aligned}
& \mathcal{L}_{I}(s)= \\
& e^{-s \sigma^{2} / P} \exp \left(-2 \pi \lambda a \int_{h}^{d}\left(1-\left(1+\frac{s r^{-\eta}}{m}\right)^{-m}\right) r d r\right) .
\end{aligned}
$$

One can infer from Theorem 1 that $P_{\mu}^{s}$ depends on the ALOHA transmission probability $a$, which should be selected carefully to maximize $P_{\mu}^{s}$. In conventional slotted ALOHA design, which is based on a collision model, ${ }^{2}$ the probability of transmission is selected such that there is only one transmission on average at every time slot. Therefore, if $N$ nodes are contending to access the spectrum, the collision based ALOHA design selects $a=1 / \mathrm{N}$ to minimize the collision probability. The spectral efficiency of such strategy is given by $e^{-1} \approx 36.8 \%$. Note that such average single transmission per time slot may lead to a conservative channel access scheme, where several time slots may be left idle (i.e., with no transmissions). According to the considered SINR capture model, successful transmission occurs as long as $\operatorname{SINR} \geq \beta$ is satisfied, which can better utilize each time slot (i.e., decrease the probability of having idle time slots) and tolerate several simultaneous transmissions.

For the SINR capture model with a given threshold $\beta$, the success probability $P_{\mu}^{s}$ monotonically increases with $a$ up to a certain saddle point, then it starts to monotonically decrease as the SINR constantly degrades by allowing more transmissions. This saddle point depends on the selected $\beta$ and can be shown to satisfy

$$
a^{*}=\frac{1}{2 \pi \lambda \int_{h}^{d}\left(1-\left(1+\frac{s r^{-\eta}}{m}\right)^{-m}\right) r d r}
$$

which follows from the first derivative test $\frac{\partial P_{\mu}^{s}}{\partial a}=0$. Before proceeding to derive the hovering time, we must note that the optimal solution of $\boldsymbol{P}_{\mathbf{1}}$ always requires (3a) to hold with equality because non-strictly satisfying (3a) (having more than $\zeta$ average successful samples) consistently requires a longer hovering time. Also considering the homogeneity of the PPP and equivalence of circular sub-regions, the drone must receive an average of $\mathbb{E}\left\{K_{\mu}\right\}=\zeta / M$ successful transmission at each hovering epoch. Hence, in order to guarantee $\mathbb{E}\left\{K_{\mu}\right\}=\zeta / M$, the drone must hover for a duration of $J_{\mu}$ time slots satisfying

$$
J_{\mu}=\frac{\mathbb{E}\left\{K_{\mu}\right\}}{P_{\mu}^{s}}=\frac{\zeta}{M P_{\mu}^{s}} .
$$

The time slot duration is designed in accordance with the information rate and the packet size as follows

$$
\tau=\frac{S}{C}=\frac{S}{B \log _{2}(1+\beta)},
$$

where $S$ is the packet size, $B$ is the bandwidth, and $C$ is the Shannon capacity of the G2A channels. Collecting these expressions, we finally arrive at the following expression for the hovering time,

$$
T_{\text {hover }}^{\mu}=J_{\mu} \tau=\frac{\zeta S}{M P_{\mu}^{s} B \log _{2}(1+\beta)} .
$$

${ }^{2}$ Collision models considers all simultaneous transmissions to be erroneous irrespective of the nodes relative channel gains. In contrast, the considered SINR capture model consider transmission success if the SINR at the receiver is greater than the threshold $\beta$ irrespective of the number of interfering nodes. 
Note that $\beta$ needs to be optimized to minimize $T_{\text {hover }}^{\mu}$. As $\beta$ increases, the channel capacity increases but $P_{\mu}^{s}$ decreases. The optimal $\beta$ value is found by solving $\frac{\partial P_{\mu}^{s}}{\partial \beta}=0$ which can only be done numerically.

\section{Traveling Time: $T_{\text {travel }}$}

Traveling time minimization problem can be further decoupled into two sub-problems: a coverage problem and a trajectory optimization problem. For a given $M$, the coverage problem seeks the minimum radius of the circles $R$ and their locations such that the union of these circles completely covers a predefined plane. The coverage problem can be solved in two concatenated levels [19]: On the inner level, $R$ is kept constant and centers of the $M$ circles are optimized to minimize the uncovered area of the sensor network. The outer level tunes $R$ whether a coverage is obtained on the inner level or not. Since the area of interest is not a constantly changing parameter, an offline table can be created for $R$ and corresponding circle locations.

Given the HLs by coverage problem, the next step is to find the optimal flight path which minimizes the total traveling time. Indeed, the flight path can be formulated as the well-known Traveling Salesman Problem (TSP) which finds the shortest possible route that visits each $\mathrm{HL}$ and returns back to the DCS [20], [21]. For the optimal flight route $Z^{*}$, the total flight distance is given by

$$
u=\sum_{\mu=1}^{M} u_{\mu}=\sum_{\mu=1}^{M}\left\|\mathbf{L}_{\mu}-\mathbf{e}_{\mu}^{T} \mathbf{Z}^{*} \mathbf{L}\right\|_{2}
$$

where $u_{\mu}$ is the distance for the $\mu^{t h}$ hop of the path, $\|\cdot\|_{2}$ denotes the $\ell_{2}$ norm, and $\mathbf{e}_{\mu} \in\{0,1\}^{M}$ is the vector with 1 in the $\mu$-th entry and 0 in the rest of the entries. Using the acceleration laws, the time needed to travel a distance $u_{\mu}$ is expressed as

$$
\tau_{\mu}=\left\{\begin{array}{ccc}
\sqrt{\frac{u_{\mu}}{\hat{q}+\check{q}},}, & \text { if } & u_{\mu} \leq \hat{u}+\check{u}, \\
\hat{t}+\check{t}+\frac{u_{\mu}-\check{u}-\check{u}}{v}, & \text { if } & u_{\mu}>\hat{u}+\check{u},
\end{array}\right.
$$

where $\hat{t}=v / \hat{q}$ and $\check{t}=v / \check{q}$ are the times needed for the UAV to change its speed from $0 \rightarrow v$ and from $v \rightarrow 0$, respectively. Similarly, $\hat{u}=1 /{ }_{2} \hat{q} \hat{t}^{2}$ and $\check{u}=1 /{ }_{2} \breve{q}^{2}$ are the required minimum distances for the drone to change its speed from $0 \rightarrow v$ and from $v \rightarrow 0$, respectively. Finally, the overall travel time can be expressed as

$$
T_{\text {travel }}=\sum_{\mu=1}^{M} \tau_{\mu}+M t_{\text {conf }},
$$

where $t_{\text {conf }}$ is an extra time needed at each stop point for configurations after arrival and before departure, respectively.

It is important to note that the coverage problem is the main sub-problem which couples the hovering and traveling time. Therefore, for a given $(M, R, \boldsymbol{L})$, the hovering time and traveling time are two independent (decoupled) problems which do not influence each other. Since $\mathcal{A}$ is constant, it is
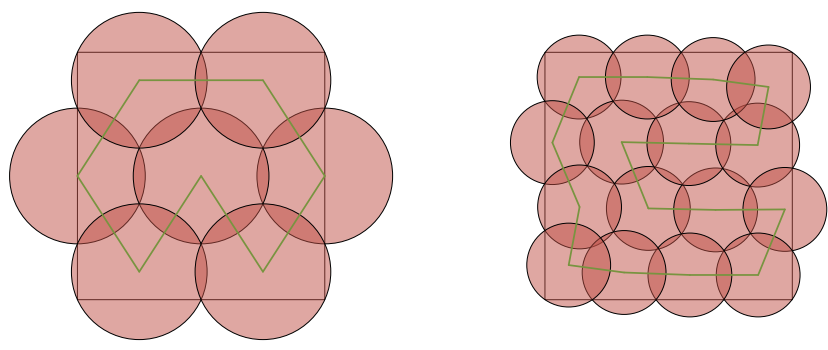

Fig. 2: Optimal covered areas radius $(R)$, HLs $(\mathbf{L})$ and trajectory for $M=7,16$.

quite practical to create an offline table from which one can find HLs, distances, and travel times for a certain range of $M$. This technique simplifies the search for the optimal $M$ resulting in a minimum total hovering and traveling time. For example, Fig. 2 demonstrates the optimal solutions for coverage and TSP problems with $M=7$ and $M=16$. Similarly, Table I shows the minimum radius of the hovering areas and the minimum distance to connect HLs given $M$.

TABLE I: Obtained $R$ and $u$ values for $M \in[1,16]$ and a network size of $\mathcal{A}=100 \times 100 \mathrm{~m}^{2}$.

\begin{tabular}{|l|l|l||l|l|l||l|l|l||l|l|l|}
\hline$M$ & $R$ & $u$ & $M$ & $R$ & $u$ & $M$ & $R$ & $u$ & $M$ & $R$ & $u$ \\
\hline \hline 1 & 70.7 & 0 & 5 & 32.6 & 223 & 9 & 23.0 & 317 & 13 & 19.4 & 396 \\
\hline 2 & 55.9 & 100 & 6 & 29.8 & 235 & 10 & 21.8 & 359 & 14 & 18.5 & 405 \\
\hline 3 & 50.3 & 161 & 7 & 27.4 & 326 & 11 & 21.2 & 337 & 15 & 17.9 & 414 \\
\hline 4 & 35.3 & 200 & 8 & 26.0 & 258 & 12 & 20.2 & 356 & 16 & 16.9 & 426 \\
\hline
\end{tabular}

\section{NUMERICAL RESUlTS}

This section first verifies the developed mathematical model against independent Monte Carlo simulations. Then, a design example is presented where the total data collection time is minimized. Throughout the simulations, we consider a PPP with density of $\lambda=0.1$ node $/ \mathrm{m}^{2}$ that is distributed over a network area of $\mathcal{A}=100 \times 100 \mathrm{~m}^{2}$. Unless otherwise stated, we use the default system parameters shown in Table II.

TABLE II: Default system parameters.

\begin{tabular}{|l|l||l|l||l|l|}
\hline Par. & Value & Par. & Value & Par. & Value \\
\hline \hline$P$ & $-30 \mathrm{dBm}$ & $\sigma^{2}$ & $-80 \mathrm{dBm}$ & $\phi$ & $90^{\circ}$ \\
\hline$\eta$ & 3 & $B$ & $200 \mathrm{KHz}$ & $S$ & $5 \mathrm{~KB}$ \\
\hline$m$ & $\{1,2,3\}$ & $v$ & $20 \mathrm{~km} / \mathrm{h}$ & $\hat{q}(\check{q})$ & $10 \mathrm{~km} / \mathrm{h}^{2}$ \\
\hline$\lambda$ & 0.1 & $\zeta$ & 250 & $\mathcal{A}$ & $10^{2} \times 10^{2} \mathrm{~m}^{2}$ \\
\hline
\end{tabular}

Consider a specific hovering area which is defined by $R=$ $h=20 \mathrm{~m}$. Fig. 3 shows the success probability $P_{\mu}^{s}$ and the throughput $P_{\mu}^{s} \log _{2}(1+\beta)$ versus $\beta$ (i.e., transmission rates) for the optimal transmission probability $a^{*}$ and the conventional ALOHA transmission probability $a=\frac{1}{\bar{N}_{\mu}}$ [22], where $\bar{N}_{\mu}=\lambda \pi R^{2}$ is the average number of nodes in $\mathcal{A}_{\mu}$.

The proposed optimal transmission probability $a^{*}$ is shown to deliver a superior performance when compared to the conventional collision based ALOHA design. However, the performance of the two schemes converges after a 


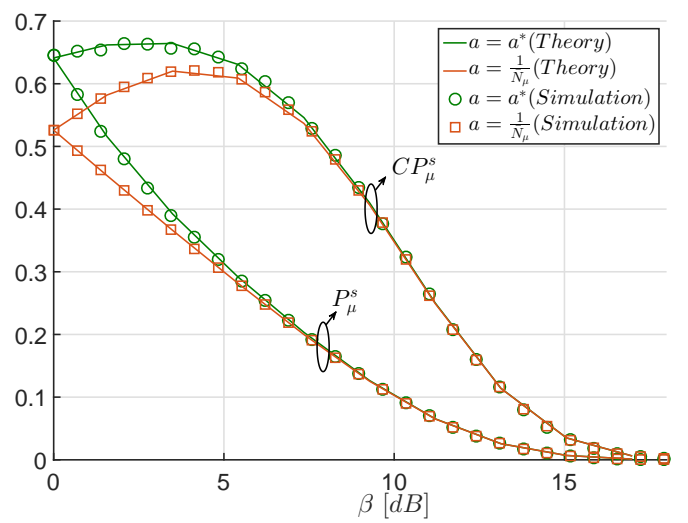

Fig. 3: $P_{\mu}^{s}$, and $P_{\mu}^{s} \log _{2}(1+\beta)$ for $a=1 / \bar{N}_{\mu}$ and $a=a^{*}$.

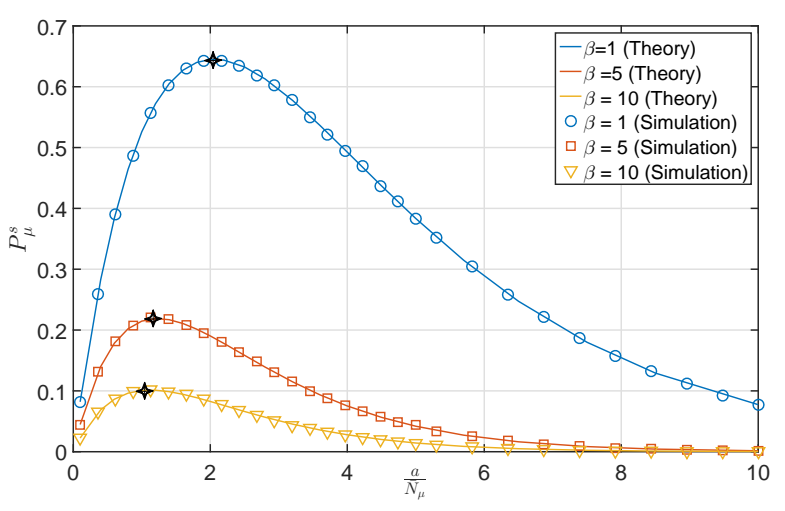

Fig. 4: $P_{\mu}^{s}$ vs. $a$ for $\beta \in\{1,5,10\}$.

certain $\beta$. This is because high values of $\beta$ implies that any interference will lead to failure, and hence, an average of a single transmission per time slot is desirable. However, this comes at the expense of being conservative and having underutilized time slots with no transmissions.

A similar discussion also applies in Fig. 4 where the success probability $P_{\mu}^{s}$ is plotted against the ALOHA transmission probability $a$. Fig. 4 shows that increasing $a$ improves the success probability up to a certain extent as the time slots are better utilized with diversified transmissions. However, increasing $a$ beyond an optimal utilization point leads to an aggressive transmission scheme where interference dominates the failure probability. This argumentation is verified by considering different $\beta$ cases: As $\beta$ increases the saddle point of $a$ decreases.

The hovering and traveling time dilemma is visualized in Fig. 5 where $P_{\mu}^{s}$ and $T_{\text {hover }}$ are plotted versus the radius $R$, i.e., $\left|\mathcal{A}_{\mu}\right|$. For given $\beta$ and $a$, increasing the hovering area drastically reduces $P_{\mu}^{s}$, which directly increases the hovering time as the drone needs to wait for a longer time duration to collect an average of $\zeta / M$ successful samples. Fig. 3, 4 and 5 show identical matching between the theoretical and simulation results, which validates the analysis.

The impact of $M$ on $T_{\text {total }}, T_{\text {hover }}$, and $T_{\text {travel }}$ are demonstrated in Fig 6. As explained before, the hovering time monotonically decreases with the number of HLs, $M$. Interestingly, the drop is considerable for small values of $M$, then it saddles on a gradual level after $M \geq 4$. In particular,

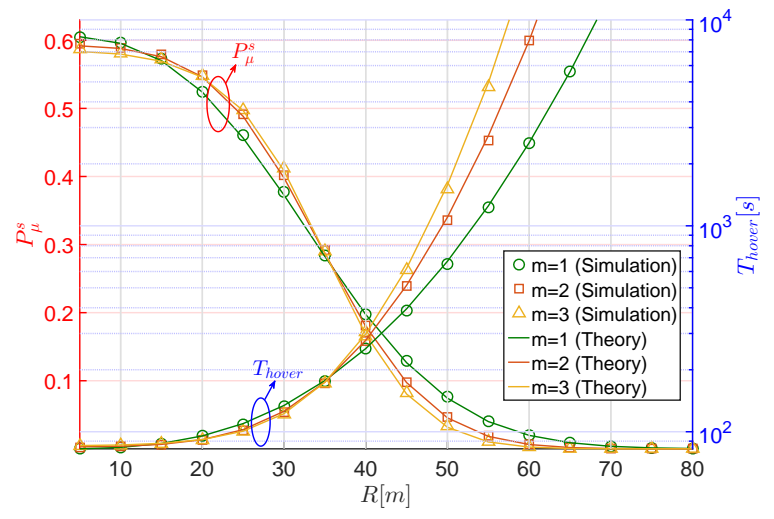

Fig. 5: $P_{\mu}^{s}$ and $T_{\text {hover }}$ vs. increasing sub-region radius, $R$.

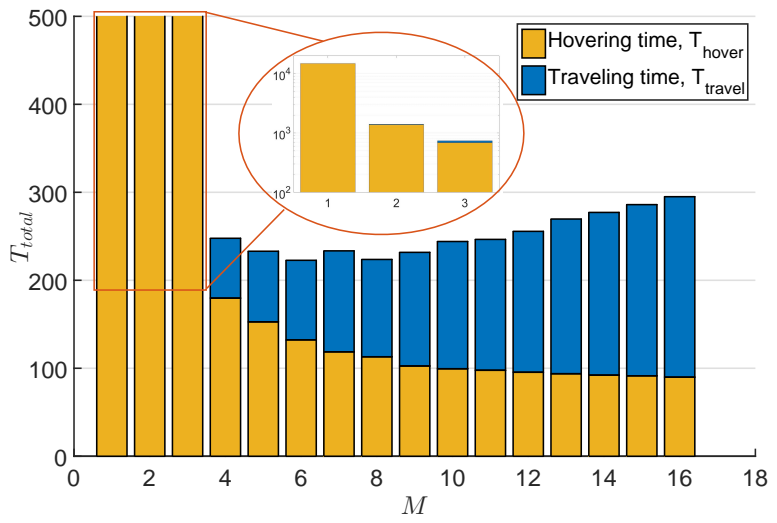

Fig. 6: $T_{\text {total }}, T_{\text {hover }}$ and $T_{\text {travel }}$ vs. the number of HLs, $M$.

the significantly sharp reduction at $M=4$ can be understood by crosschecking Table II and Fig. 5. On the other hand, traveling time increase with $M$ starts playing an important role especially after $M \geq 4$. The total traveling time is determined based on $T_{\text {hover }}$, and $T_{\text {travel }}$ and is shown to achieve its minimum at $M=6$ with $T_{\text {total }}=223 \mathrm{~s}$. We must note that the optimal point of $T_{\text {total }}$ is subject to different system parameters such as $\zeta, \beta, S, B$, etc.

\section{CONCLUSION}

This paper proposes aerial data aggregation from a finite spatial field via an UAV. The objective is to collect an average number of samples from wireless nodes, that are spatially scattered according to a Poisson point process, within minimal time. The field is divided into several subregions. An UAV with wireless capabilities sequentially hover over each subregion to collect data from the underlying nodes via slotted ALOHA protocol. To this end, an optimization problem is formulated and solved to find the optimal number of subregions, the area of each subregion, the hovering locations, the hovering time, and the trajectory between hovering locations such that an average number of samples are collected in minimal time. The proposed formulation is shown to be $n p$ hard mixed integer problem, and hence, a decoupled heuristic solution is proposed. Particularly, stochastic geometry is used to find the optimal channel access probability such that the average number of samples are collected from each subregion 
in minimal hovering time. The number and locations of the hovering spots are found by solving a coverage problem. The trajectory between the hovering locations is obtained by solving a travel salesman problem. The results show that there exist an optimal solution that balance the tradeoff between the hovering and traveling times such that the total time for collecting the required samples is minimized.

\section{APPENDIX A \\ PROOF OF THEOREM 1}

We start by deriving the Laplace transform of the normalized interference plus noise power distribution,

$$
\begin{aligned}
& \mathcal{L}_{I}(s) \stackrel{(a)}{=} \mathbb{E}_{I}\left[e^{-s I}\right] \stackrel{(b)}{=} \mathbb{E}_{D} \mathbb{E}_{G}\left[e^{-s \sigma^{2} / P} \prod_{\mathbf{x} \in \tilde{\mathbf{\Psi}}_{\mu} \backslash \mathbf{x}_{\mathbf{n}}} e^{-s G_{x} D_{x}^{-\eta}}\right] \\
& \stackrel{(c)}{=} e^{-s \sigma^{2} / P} \mathbb{E}_{D}\left[\prod_{\mathbf{x} \in \tilde{\mathbf{\Psi}}_{\mu} \backslash \mathbf{x}_{\mathbf{n}}}\left(1+\frac{s D_{x}^{-\eta}}{m}\right)^{-m}\right] \\
& \stackrel{(d)}{=} e^{-s \sigma^{2} / P} \exp \left(-2 a \pi \lambda \int_{h}^{d}\left(1-\left(1+\frac{s r^{-\eta}}{m}\right)^{-m}\right) r d r\right)
\end{aligned}
$$

where $(a)$ follows from the definition of the Laplace transform. (b) follows from the independence between the PDFs $f_{D}\left(r_{x}\right)$ and $f_{G}(g)$. In $(c)$, since the channel gains of different nodes are i.i.d., the product of expectations $\prod \mathbb{E}_{G_{x}}(\cdot)$ is equal to the expectation of the product $\mathbb{E}_{G} \prod(\cdot)$ where $\mathbb{E}_{G_{x}}(\cdot)$ follows from the moment generating function (MGF) of gamma distribution. And $(d)$ follows from the probability generating functional (PGFL) of PPP process and the distance distribution as given in (4). To this end, the coverage probability is derived in terms of $\mathcal{L}_{I}(s)$ as follows,

$$
\begin{aligned}
& P_{\mu}^{s}=\mathbb{P}\left(\bigcup_{\mathbf{x}_{\mathbf{n}} \in \tilde{\mathbf{\Psi}}_{\mu}} \operatorname{SINR}_{\mu}^{n}>\beta\right) \\
& \stackrel{(a)}{=} \mathbb{E}_{D_{n}} \mathbb{E}_{I}\left[\sum_{\mathbf{x}_{\mathbf{n}} \in \tilde{\mathbf{\Psi}}_{\mu}} \mathbb{P}\left(G_{n}>\beta D_{n}^{\eta} I \mid D_{n}, I\right)\right] \\
& \stackrel{(b)}{=} \mathbb{E}_{D_{n}} \mathbb{E}_{I}\left[\sum_{\mathbf{x}_{\mathbf{n}} \in \tilde{\mathbf{\Psi}}_{\mu}} \frac{\Gamma\left(m, m \beta r^{\eta} I\right)}{\Gamma(m)} \mid D_{n}\right] \\
& \stackrel{(c)}{=} \mathbb{E}_{D_{n}} \mathbb{E}_{I}\left[\sum_{\mathbf{x}_{\mathbf{n}} \in \tilde{\boldsymbol{\Psi}}_{\mu}} \sum_{k=0}^{m-1} \frac{\left(m \beta r^{\eta} I\right)^{k}}{k !} e^{-m \beta r^{\eta} I} \mid D_{n}\right] \\
& \stackrel{(d)}{=} 2 a \pi \lambda \int_{h}^{d} \sum_{k=0}^{m-1} \frac{\left(-m \beta r^{\eta}\right)^{k}}{k !}\left[\frac{\partial^{k}}{\partial s^{k}} \mathcal{L}_{I}(s)\right]_{s=m \beta r^{\eta}} r d r
\end{aligned}
$$

where in $(a)$ the union is equivalent to summation since the events are mutually exclusive, [18]. (b) follows from the $\mathrm{CCDF}$ of $G_{n}$ and that $\mathbb{P}\left(\operatorname{SINR}_{\mu}^{n}>\beta\right)$ are i.i.d over $n$. (c) follows from the incomplete gamma function definition for $m \in \mathbb{Z}^{+} .(d)$ follows from Campbell Mecke Theorem [23].

\section{REFERENCES}

[1] A. Al-Fuqaha, M. Guizani, M. Mohammadi, M. Aledhari, and M. Ayyash, "Internet of things: A survey on enabling technologies, protocols, and applications," IEEE Communications Surveys Tutorials, vol. 17, no. 4, pp. 2347-2376, Fourthquarter 2015.
[2] J. N. Al-Karaki and A. E. Kamal, "Routing techniques in wireless sensor networks: a survey," IEEE Wireless Communications, vol. 11, no. 6, pp. 6-28, Dec 2004.

[3] Y. Zeng, R. Zhang, and T. J. Lim, "Wireless communications with unmanned aerial vehicles: opportunities and challenges," IEEE Communications Magazine, vol. 54, no. 5, pp. 36-42, May 2016.

[4] B. V. D. Bergh, A. Chiumento, and S. Pollin, "LTE in the sky: trading off propagation benefits with interference costs for aerial nodes," IEEE Communications Magazine, vol. 54, no. 5, pp. 44-50, May 2016.

[5] A. Merwaday, A. Tuncer, A. Kumbhar, and I. Guvenc, "Improved throughput coverage in natural disasters: Unmanned aerial base stations for public-safety communications," IEEE Vehicular Technology Magazine, vol. 11, no. 4, pp. 53-60, Dec 2016.

[6] R. Sun and D. W. Matolak, "Initial results for airframe shadowing in Land C-band air-ground channels," in 2015 Integrated Communication, Navigation and Surveillance Conference (ICNS), April 2015.

[7] D. W. Matolak and R. Sun, "Unmanned aircraft systems: Air-ground channel characterization for future applications," IEEE Vehicular Technology Magazine, vol. 10, no. 2, pp. 79-85, June 2015.

[8] A. Al-Hourani, S. Kandeepan, and A. Jamalipour, "Modeling air-toground path loss for low altitude platforms in urban environments," in 2014 IEEE Global Communications Conference, Dec 2014.

[9] A. Al-Hourani, S. Kandeepan, and S. Lardner, "Optimal LAP altitude for maximum coverage," IEEE Wireless Communications Letters, vol. 3, no. 6, pp. 569-572, Dec 2014.

[10] A. AL-Hourani, S. Chandrasekharan, G. Kaandorp, W. Glenn, A. Jamalipour, and S. Kandeepan, "Coverage and rate analysis of aerial base stations [letter]," IEEE Transactions on Aerospace and Electronic Systems, vol. 52, no. 6, pp. 3077-3081, December 2016.

[11] V. V. Chetlur and H. S. Dhillon, "Downlink coverage analysis for a finite 3-D wireless network of unmanned aerial vehicles," IEEE Transactions on Communications, vol. 65, no. 10, pp. 4543-4558, Oct 2017.

[12] M. Mozaffari, W. Saad, M. Bennis, and M. Debbah, "Wireless communication using unmanned aerial vehicles (UAVs): Optimal transport theory for hover time optimization," IEEE Transactions on Wireless Communications, vol. 16, no. 12, pp. 8052-8066, Dec 2017.

[13] _ "Mobile unmanned aerial vehicles (UAVs) for energy-efficient internet of things communications," IEEE Transactions on Wireless Communications, vol. 16, no. 11, pp. 7574-7589, Nov 2017.

[14] A. Giorgetti, M. Lucchi, M. Chiani, and M. Z. Win, "Throughput per pass for data aggregation from a wireless sensor network via a UAV," IEEE Transactions on Aerospace and Electronic Systems, vol. 47, no. 4, pp. 2610-2626, OCTOBER 2011.

[15] R. Sugihara and R. K. Gupta, "Optimal speed control of mobile node for data collection in sensor networks," IEEE Transactions on Mobile Computing, vol. 9, no. 1, pp. 127-139, Jan 2010.

[16] D. Wackerly, W. Mendenhall, and R. Scheaffer, Mathematical Statistics with Applications. Pacific Grove CA, USA: Duxbury, 2002.

[17] M. K. Simon and M.-S. Alouini, Digital communication over fading channels. John Wiley \& Sons, 2005, vol. 95.

[18] H. S. Dhillon, R. K. Ganti, F. Baccelli, and J. G. Andrews, "Modeling and analysis of k-tier downlink heterogeneous cellular networks," IEEE Journal on Selected Areas in Communications, vol. 30, no. 3, pp. 550-560, April 2012.

[19] K. J. Nurmela and P. R. J. Östergård, "Covering a square with up to 30 equal circles," 2000.

[20] M. Dorigo and L. M. Gambardella, "Ant colony system: a cooperative learning approach to the traveling salesman problem," IEEE Transactions on Evolutionary Computation, vol. 1, no. 1, pp. 53-66, Apr 1997.

[21] J. Kirk, "Traveling salesman problemgenetic algorithm, version $1.3 \quad$ (2014)," https://www.mathworks.com/matlabcentral/fileexchange/13680traveling-salesman-problem-genetic-algorithm, MATLAB Central File Extchange, Retrieved, Jan. 2018.

[22] L. G. Roberts, "Aloha packet system with and without slots and capture," SIGCOMM Comput. Commun. Rev., vol. 5, no. 2, pp. 28-42, Apr. 1975.

[23] S. N. Chiu, D. Stoyan, W. S. Kendall, and J. Mecke, Stochastic geometry and its applications. John Wiley \& Sons, 2013. 